\title{
Core excitation in three-body nuclear reactions: Improved nucleon-core potential
}

\author{
A. Deltuva* \\ Institute of Theoretical Physics and Astronomy, Vilnius University, A. Goštauto 12, LT-01108 Vilnius, Lithuania
}

(Received December 22, 2014)

\begin{abstract}
Three-body nuclear reactions in two-nucleon plus core systems are described in the framework of exact scattering equations including the core excitation. A nucleon-core optical potential is constructed that can be easily adjusted to the reference potential and thereby to the experimental two-body data, if available. This constitutes an important improvement over the simple deformation of the potential used previously that violated the original fit to the data. Predictions for elastic, inelastic, and transfer reactions involving ${ }^{10} \mathrm{Be}$ and ${ }^{24} \mathrm{Mg}$ nuclear cores are obtained. The new optical potential leads to a moderate increase of cross sections.
\end{abstract}

PACS numbers: 24.10.-i, 21.45.-v, 25.45.Hi, 25.40.Hs

\section{INTRODUCTION}

Deuteron $(d)$ scattering from a nucleus $A$, consisting of $A$ nucleons, microscopically is an $A+2$ body problem. While various $A \geq 3$ cases have been considered using approximate methods 1], a rigorous solution of exact scattering equations 2 [5] has been achieved so far for three- and four-body systems only [6 12]. In addition, there exist also numerous approximate treatments for three-body reactions [13 15]. To apply the available three-body techniques to deuteron-nucleus scattering, this process is often approximated by a three-body problem where the nuclear core $A$ is treated as a structureless particle whose interaction with the proton $(p)$ and the neutron $(n)$ is given by complex or real potentials. In some cases this may be a reasonable approach, but in others it is necessary to go beyond the picture of an inert core $A$ and take into account its internal degrees of freedom. It has been shown in several works $16-19]$ that the excitation of the core may be an important reaction mechanism and needs to be taken into account. There are several formulations of rigorous three-body scattering equations including core excitation (CeX) [19, 20], however, numerical results were obtained only in Ref. 19]. As dynamic input these calculations use nucleon-nucleon $(N N)$ and nucleon-core $(N A)$ interactions. There is a number of standard parametrizations for $N A$ optical potentials (OP) without CeX. The CeX is usually included by deforming these standard potentials to allow the coupling between ground $(A)$ and excited $\left(A^{*}\right)$ states of the core. However, this way the additional $N A^{*}$ component in the two-body scattering equation together with the potential deformation distort the elastic $N A$ amplitude that deviates from the original elastic amplitude calculated using standard potential without $\mathrm{CeX}$. The latter is usually fitted to the $A(N, N) A$ elastic experimental data, thus, the description of the data gets destroyed when including the $\mathrm{CeX}$ via the simple deformation of the potential. Furthermore, if the $N A-N A^{*}$ coupling potential was fitted to the inelastic $A\left(N, N^{\prime}\right) A^{*}$ data using

\footnotetext{
* arnoldas.deltuva@tfai.vu.lt
}

the distorted-wave Born approximation (DWBA) which is the usual case, the fit to $A\left(N, N^{\prime}\right) A^{*}$ data is lost as well. Thus, an additional adjustment of the OP parameters needs to be performed to restore the description of $N A$ elastic and inelastic scattering data. This implies a new fit procedure for each nucleus at each energy, which may be quite tedious. On the other hand, one may take the advantage of already existing fits to the experimental data obtained using DWBA and standard OP. For this a $N A$ potential with $\mathrm{CeX}$ is needed that, when inserted into the exact two-body multichannel LippmannSchwinger equation, yields the standard OP results for the $A(N, N) A$ elastic scattering and DWBA results for the $A\left(N, N^{\prime}\right) A^{*}$ reaction. In the present work I propose a method for achieving this goal, i.e., I construct a $N A$ potential with $\mathrm{CeX}$ that at a given energy exactly reproduces the standard OP and DWBA amplitudes for $A(N, N) A$ and $A\left(N, N^{\prime}\right) A^{*}$ scattering processes, respectively. Thus, the desired consistency with the $N A$ experimental data is ensured. In case there are no data available, this approach still allows for a more precise evaluation of the CeX effect, not affected by the OP mismatch. In three-body systems I aim to study the changes in observables caused by this improvement of the OP. I therefore reanalyze the reactions calculated in Ref. 19] using the simple deformation of the OP as well as present several new cases.

In Sec. III I derive the $N A$ potential with $\mathrm{CeX}$, and in Sec. IIII I recall the three-body scattering theory. Example results for $d+{ }^{10} \mathrm{Be}, p+{ }^{11} \mathrm{Be}$, and $d+{ }^{24} \mathrm{Mg}$ reactions are presented in Sec. IV] while the summary is given in Sec. V.

\section{SUBTRACTION METHOD FOR NUCLEON-CORE POTENTIAL}

I employ the extended Hilbert space $\mathcal{H}_{g} \oplus \mathcal{H}_{x}$ where the two sectors correspond to the core being in its ground (g) or excited (x) state [19]. I aim to construct the $N A$ potential coupling the two sectors and denote its components by $V_{g g}, V_{x x}$, and $V_{x g}=V_{g x}^{T}$; they are represented graphically in Fig. 1] The coupled-channel Lippmann- 


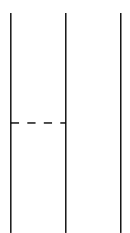

(a)

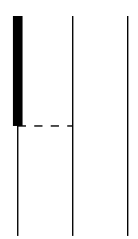

(b)

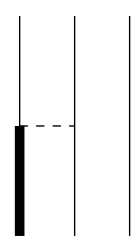

(c)

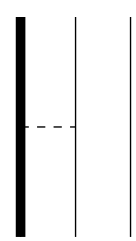

(d)

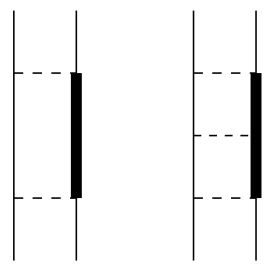

FIG. 2. Diagrammatic representation of the contributions at the two lowest orders subtracted from the nucleon-core potential. space. The particles are represented by vertical lines, the core in its excited state is distinguished by thick lines, the potential by horizontal dashed lines. The diagrams (a), (b), (c), and (d) correspond to $V_{g g}, V_{x g}, V_{g x}$, and $V_{x x}$.

Schwinger equation

$$
\left(\begin{array}{l}
T_{g g} T_{g x} \\
T_{x g} T_{x x}
\end{array}\right)=\left(\begin{array}{l}
V_{g g} V_{g x} \\
V_{x g} V_{x x}
\end{array}\right)+\left(\begin{array}{c}
V_{g g} V_{g x} \\
V_{x g} V_{x x}
\end{array}\right) G_{0}\left(\begin{array}{c}
T_{g g} T_{g x} \\
T_{x g} T_{x x}
\end{array}\right)
$$

yields the respective components $T_{j i}$ of the $N A$ transition matrix at the available energy $E$ with $G_{0}=$ $\left(E+i 0-H_{0}\right)^{-1}$ being the free resolvent. I emphasize that the extended free Hamiltonian $H_{0}$ besides the kinetic energy operator contains also the internal core Hamiltonian whose contribution vanishes for $\mathcal{H}_{g}$ but is equal to the core excitation energy $\left(m_{A^{*}}-m_{A}\right)$ for $\mathcal{H}_{x}$.

At energy $E=E_{s}$ the component $T_{g g}$ describing the $N A$ elastic scattering is demanded to reproduce the transition matrix

$$
t_{g}=v_{g}+v_{g} G_{0} t_{g}
$$

obtained with the $N A$ potential $v_{g}$ not including CeX, usually taken from one of the standard parametrizations. There is no such demand for $T_{x x}$ due to the lack of the experimental data for the $N A^{*}$ elastic scattering. The respective transition matrix for the core in its excited state but without coupling to the ground state

$$
t_{x}=v_{x}+v_{x} G_{0} t_{x}
$$

is obtained with the $N A^{*}$ potential $v_{x}$ that is not constrained by the data but is usually taken from the same standard OP parametrization as $v_{g}$.

Furthermore, at $E=E_{s}$ I also require $T_{x g}$ to reproduce the DWBA amplitude

$$
T_{x g}^{\mathrm{DWBA}}=\left(1+t_{x} G_{0}\right) V_{x g}^{\mathrm{DWBA}}\left(1+G_{0} t_{g}\right) .
$$

The potential $V_{x q}^{\text {DWBA }}$ coupling the two Hilbert sectors $\mathcal{H}_{g}$ and $\mathcal{H}_{x}$ usually is obtained by deforming the central part of $v_{g}$. In the rotational model [21] one assumes that the core has a permanent quadrupole deformation and replaces in $v_{g}$ the nuclear radius $R_{0}$ by $R=R_{0}\left(1+\beta_{2} Y_{20}(\hat{\xi})\right)$, where $\beta_{2}$ is the quadrupole deformation parameter and $\hat{\xi}$ describes the internal core degrees of freedom in the body-fixed frame. If the central part of $v_{g}$ is a function of $\left(r-R_{0}\right)$, e.g., the WoodsSaxon function, $V_{x g}^{\mathrm{DWBA}}$ is given by $\mathcal{P}_{x} v_{g}\left(r-\delta_{2} Y_{20}(\hat{\xi})\right) \mathcal{P}_{g}$ where $\mathcal{P}_{i}$ are the respective projectors and $\delta_{2}=\beta_{2} R_{0}$ is the deformation length.

To fulfill the above demands, $T_{x g}$ in Eq. (11) is resolved as

$$
T_{x g}=\left(1-V_{x x} G_{0}\right)^{-1} V_{x g}\left(1+G_{0} T_{g g}\right)
$$

and used in this for the $T_{g g}$ component, leading to

$T_{g g}=V_{g g}\left(1+G_{0} T_{g g}\right)+V_{g x} G_{0}\left(1-V_{x x} G_{0}\right)^{-1} V_{x g}\left(1+G_{0} T_{g g}\right)$.

Furthermore, I make use of the identity

$$
\left(1-v_{x} G_{0}\right)^{-1}=1+t_{x} G_{0}
$$

obtained from Eq. (3). By comparing Eqs. (6) and (5) with (2) and (4) one observes that the conditions $T_{g g}=t_{g}$ and $T_{x g}=T_{x g}^{\mathrm{DWBA}}$ are satisfied at $E=E_{s}$ by choosing

$$
\begin{aligned}
V_{g g} & =v_{g}-V_{g x} g_{0}^{s}\left(1+t_{x} g_{0}^{s}\right) V_{x g}, \\
V_{x g} & =V_{x g}^{\mathrm{DWBA}} \\
V_{x x} & =v_{x}
\end{aligned}
$$

with $g_{0}^{s}=\left(E_{s}+i 0-H_{0}\right)^{-1}$. Thus, the essential idea of the present method is subtracting from the elastic amplitude the contributions that are explicitly generated in the scattering equations by the coupling to the core excited state. For this reason it is called the subtraction method. The subtracted contributions at the two lowest orders are diagrammatically represented in Fig. 2, These contributions are nonlocal in the coordinate space but this has no disadvantage when the calculations are performed in the momentum space.

Note that a similar subtraction method has been used in the past to readjust the purely nucleonic part of the two-nucleon potential with explicit $\Delta$-isobar excitation [22], thereby improving the fit of the elastic two-nucleon scattering data. The present method is more general in the sense that it also fixes the inelastic amplitude.

\section{THREE-PARTICLE SCATTERING EQUATIONS}

Exact three-body scattering equations of Faddeev or Alt-Grassberger-Sandhas (AGS) type including the CeX 
have been discussed in Refs. [19, 20]; their practical solution was implemented in Ref. [19]. In the extended Hilbert space they acquire the standard form of the AGS integral equations for three-body transition operators

$$
U_{\beta \alpha}=\bar{\delta}_{\beta \alpha} G_{0}^{-1}+\sum_{\gamma=1}^{3} \bar{\delta}_{\beta \gamma} T_{\gamma} G_{0} U_{\gamma \alpha}
$$

that couple the two sectors $\mathcal{H}_{g}$ and $\mathcal{H}_{x}$ much like the two-body transition operators $T_{\gamma}$ in Eq. (11). The subscripts $\alpha, \beta, \gamma$ label the spectator particles (interacting pairs in the odd-man-out notation) for operator components, while $\bar{\delta}_{\beta \alpha}=1-\delta_{\beta \alpha}$. Following the developments of Ref. [19], I solve Eq. (9) numerically in the momentum-space partial-wave framework, including the proton-core Coulomb force via the screening and renormalization method [23 25]. The scattering amplitudes are given by the on-shell matrix elements of the transition operators $U_{\beta \alpha}$ calculated between initial and final channel states.

\section{RESULTS}

In this section I present results for two-cluster elastic, inelastic, and transfer reactions initiated by $p+{ }^{10} \mathrm{Be}$, $d+{ }^{10} \mathrm{Be}, p+{ }^{11} \mathrm{Be}$, and $d+{ }^{24} \mathrm{Mg}$ collisions. For both ${ }^{10} \mathrm{Be}$ and ${ }^{24} \mathrm{Mg}$ cores the ground (first excited) states have spin and parity $0^{+}\left(2^{+}\right)$, while the respective excitation energies are 3.368 and $1.369 \mathrm{MeV}$. The main goal is to evaluate the changes in the observables due to the use of the improved nucleon-core potential including the CeX with subtraction. I present three types of calculations. All of them use a realistic CD Bonn potential [26] for the $n p$ pair, but differ in $N A$ interactions: (i) single-particle (SP) model for the core, i.e., neglecting $\mathrm{CeX}$ in $N A$ potentials, labeled as $\mathrm{SP}$ in the following; (ii) including CeX via simple deformation of the SP potential without subtraction and readjustment, labeled CX(no subtr) in the following; and (iii) including CeX using improved $N A$ potentials with subtraction and proper readjustment as described in Sec. III labeled CX in the following. Thus, the difference between CX and SP will yield the the CeX effect, while the difference between CX and CX (no subtr) will evaluate the importance of the potential improvement. The calculations SP and CX(no subtr), both based on the Chapel Hill 89 (CH89) parametrization [27], are the same as in Ref. 19], but CX(no subtr) was labeled simply CX in Ref. 19]. I therefore only have to describe the calculations CX with subtraction. For the $n A$ pair in the partial waves with $(A n)$ bound states I take over the real binding potentials from Ref. [19] since they are already adjusted to experimental binding energies and need no subtraction. Depending on the partial wave, these potentials may support deeply bound states $\left|b_{0}\right\rangle$ that are Pauli forbidden and therefore have to be projected out; this is achieved by adding a separable term $\left|b_{0}\right\rangle \Gamma\left\langle\left|b_{0}\right|\right.$ with $\Gamma \geq 1 \mathrm{GeV}$ to

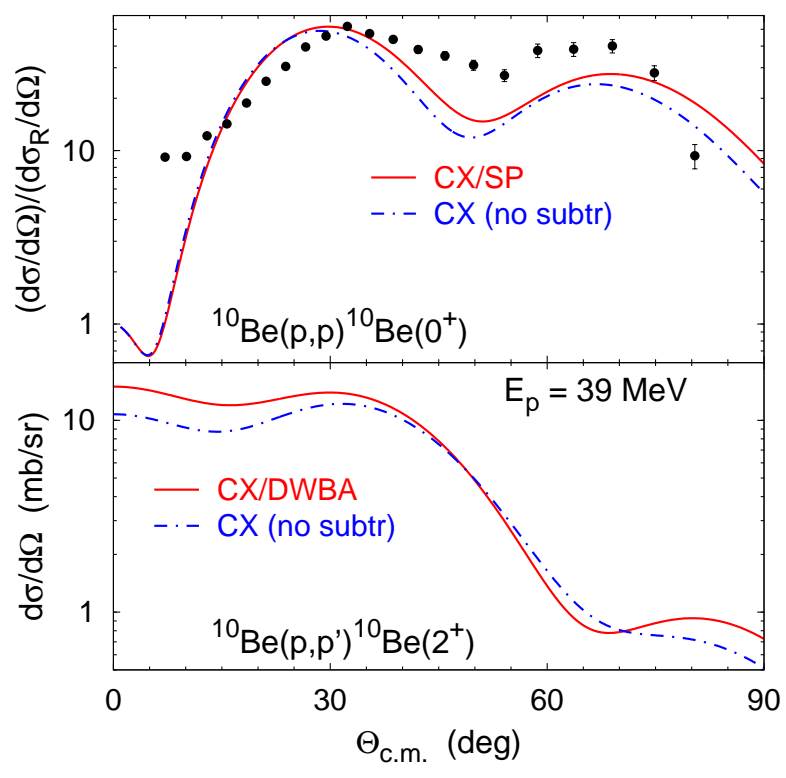

FIG. 3. (Color online) Differential cross section for ${ }^{10} \mathrm{Be}(p, p){ }^{10} \mathrm{Be}$ and ${ }^{10} \mathrm{Be}\left(p, p^{\prime}\right){ }^{10} \mathrm{Be}^{*}$ reactions at $E_{p}=39$ $\mathrm{MeV}$. Results including the CeX with and without subtraction, i.e., CX and CX(no subtr), are given by solid and dashed-dotted curves, respectively. CX predictions coincide with SP results neglecting the CeX for the elastic scattering and with DWBA results for the inelastic reaction. The elastic experimental data are from Ref. [31].

the local $n A$ potential and thereby moving the Pauli forbidden state $\left|b_{0}\right\rangle$ to a large positive energy [28]. For $n A$ in other partial waves and for $p A$ in all partial waves I take the potentials including $\mathrm{CeX}$ with subtraction; they are derived from the $\mathrm{CH} 89$ parametrization unless explicitly stated otherwise. The subtraction energy $E_{s}$ coincides with the energy at which the OP is taken, i.e., half of the deuteron energy $E_{d}$ for $n A$ but proton energy $E_{p}$ for $p A$. However, if the $n A$ potential is complex in all partial waves as in the case of the present $d+{ }^{24} \mathrm{Mg}$ calculations, the $p+(A n)$ channel is absent and therefore the $p A$ potential is taken at $E_{d} / 2$ as well. These choices are the same as in Ref. [19]. The employed quadrupole deformation parameters for $N-{ }^{10} \mathrm{Be}$ interactions are $\beta_{2}=0.67$ and $\delta_{2}=1.664 \mathrm{fm}$ as in Ref. [19], while for $N_{-}{ }^{24} \mathrm{Mg} \mathrm{I}$ use $\beta_{2}=0.5$ and $\delta_{2}=1.69 \mathrm{fm}$. In a few selected cases I present CX results derived from Watson [29] and KoningDelaroche [30] parametrizations; those calculations use the $\delta_{2}$ values listed above and are labeled as $\mathrm{CX}(\mathrm{W})$ and $\mathrm{CX}(\mathrm{KD})$, respectively.

First in Fig. 3 I demonstrate the importance of the proper potential readjustment by comparing the CX and CX(no subtr) results in the two-body system. As an example I show the differential cross section $d \sigma / d \Omega$ for elastic and inelastic $p+{ }^{10} \mathrm{Be}$ scattering at $E_{p}=39 \mathrm{MeV}$ proton energy as a function of the center-of-mass (c.m.) scattering angle $\Theta_{\text {c.m. }}$. Here and in the following the 


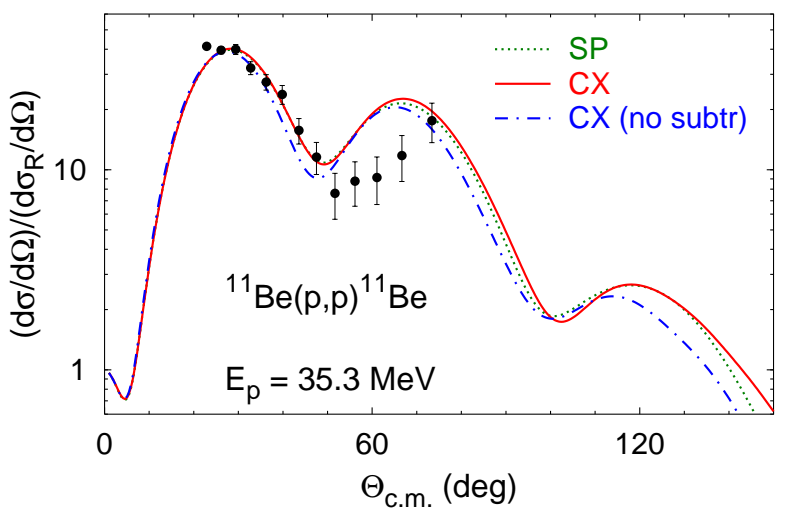

FIG. 4. (Color online) Differential cross section for $p+{ }^{11} \mathrm{Be}$ elastic scattering at $E_{p}=35.3 \mathrm{MeV}$. Results of SP, CX, and CX(no subtr) potential models are given by dotted, solid, and dashed-dotted curves, respectively. The experimental data are from Ref. [31].

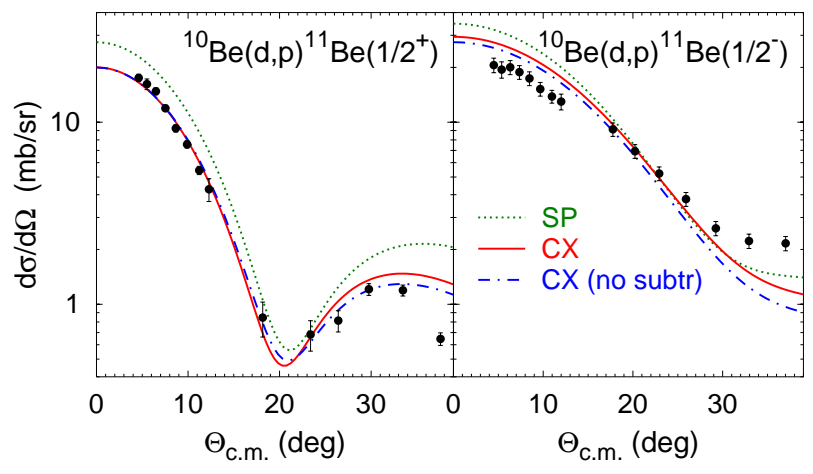

FIG. 5. (Color online) Differential cross section for transfer reactions ${ }^{10} \mathrm{Be}(d, p){ }^{11} \mathrm{Be}$ at $E_{d}=21.4 \mathrm{MeV}$ leading to the ground $\left(\frac{1}{2}^{+}\right)$and excited $\left(\frac{1}{2}^{-}\right)$states of ${ }^{11} \mathrm{Be}$. Curves are as in Fig. 4 and experimental data are from Ref. [32].

elastic differential cross section is given as a ratio to the Rutherford cross section $d \sigma_{R} / d \Omega$. The differences between the CX and CX(no subtr) calculations are of moderate size for elastic scattering but become more significant for the ${ }^{10} \mathrm{Be}\left(p, p^{\prime}\right){ }^{10} \mathrm{Be}^{*}$ reaction, reaching nearly $40 \%$ at forward angles. I remind that by construction the CX and SP results coincide for the elastic scattering while the CX and DWBA results coincide for the inelastic scattering.

Observables for $p+{ }^{10} \mathrm{Be}$ and $p+{ }^{11} \mathrm{Be}$ elastic scattering are quite strongly correlated. Consistently with this fact the SP and CX models, yielding identical results for ${ }^{10} \mathrm{Be}(p, p){ }^{10} \mathrm{Be}$, agree quite well also for ${ }^{11} \mathrm{Be}(p, p){ }^{11} \mathrm{Be}$ at $E_{p}=35.3 \mathrm{MeV}$ as shown in Fig. 4, indicating that the CeX effect on the $p+{ }^{11} \mathrm{Be}$ elastic cross section is very small. However, the $p^{-10} \mathrm{Be}$ potential, that does not properly describes elastic two-body data as in the case of CX(no subtr), overestimates the CeX effect.

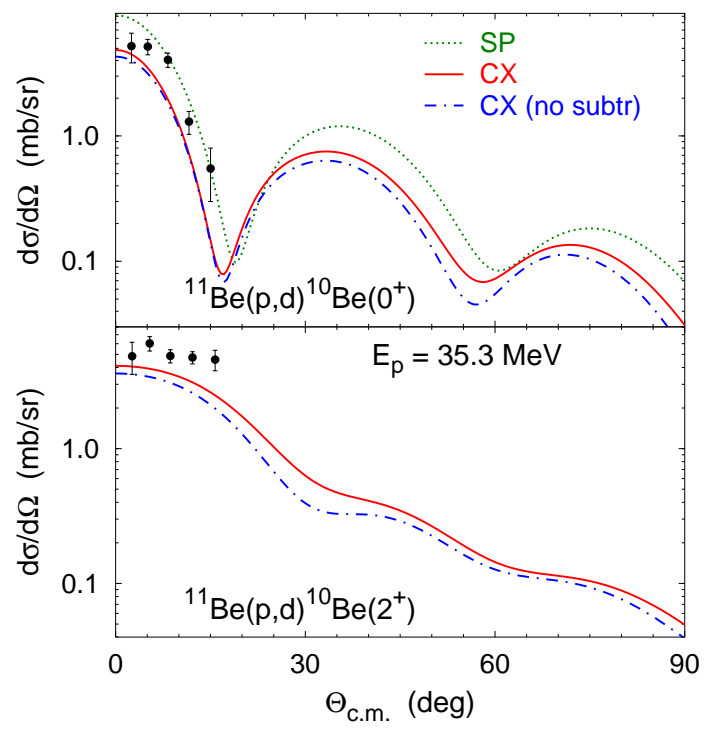

FIG. 6. (Color online) Differential cross section for ${ }^{11} \mathrm{Be}(p, d){ }^{10} \mathrm{Be}$ transfer reactions at $E_{p}=35.3 \mathrm{MeV}$ leading to the ground $\left(0^{+}\right)$and excited $\left(2^{+}\right)$states of ${ }^{10} \mathrm{Be}$. Curves are as in Fig. 4 and experimental data are from Ref. [33].

Next I show in Fig. 5 the differential cross sections for the ${ }^{10} \mathrm{Be}(d, p){ }^{11} \mathrm{Be}$ transfer reactions at $E_{d}=21.4 \mathrm{MeV}$ deuteron energy. Quite surprisingly, the improvement of the $N_{-}{ }^{10} \mathrm{Be}$ potentials has a very small effect on the transfer to the ${ }^{11} \mathrm{Be}$ ground state $\frac{1}{2}^{+}$. For the transfer to the ${ }^{11} \mathrm{Be}$ excited state $\frac{1}{2}^{-}$the effect is of moderate size but not beneficial. Thus, the ${ }^{10} \mathrm{Be}(d, p)^{11} \mathrm{Be}^{*}$ reaction still remains an unresolved problem and calls for a more sophisticated model. In Fig. 6 I present results for ${ }^{11} \mathrm{Be}(p, d){ }^{10} \mathrm{Be}$ transfer reactions at $E_{p}=35.3 \mathrm{MeV}$; it corresponds to ${ }^{10} \mathrm{Be}(d, p){ }^{11} \mathrm{Be}$ at $E_{d}=40.3 \mathrm{MeV}$. At this higher energy the importance of the proper potential adjustment is more visible, especially for the reaction leading to the $2^{+}$excited state of the ${ }^{10} \mathrm{Be}$ core. The latter finding is not unexpected given the results for $p+{ }^{10} \mathrm{Be}$ inelastic scattering in Fig. 3. For both ground and excited ${ }^{10} \mathrm{Be}$ states the description of the experimental data is improved, although some discrepancies still remain. In fact, these predictions are quite sensitive to the choice of the optical potential from which the CX model is derived. I illustrate this finding in Fig. 7 by comparing the CX results for the ${ }^{11} \mathrm{Be}(p, d){ }^{10} \mathrm{Be}$ differential cross sections based on CH89, Watson, and Koning-Delaroche OP parametrizations. I admit that no one of them reproduces the experimental data for both reactions simultaneously: transfer to the ${ }^{10} \mathrm{Be}$ ground state $0^{+}$is best described by $\mathrm{CX}(\mathrm{KD})$ while to the excited state $2^{+}$by $\mathrm{CX}(\mathrm{W})$.

In Ref. [19] it was demonstrated that the CeX effect in transfer reactions is, in general, much more complicated than just a simple rescaling of the SP differential cross section by the respective spectroscopic factor (SF). This 


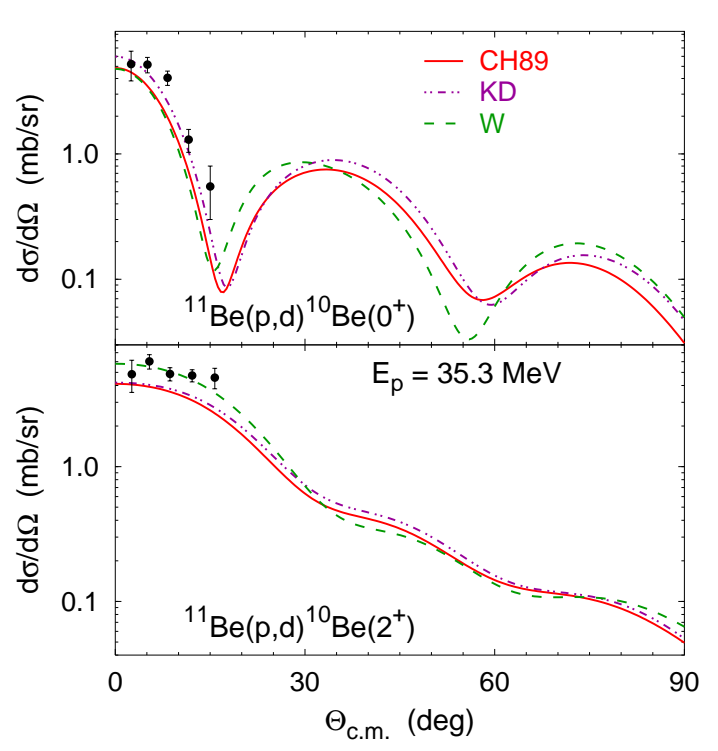

FIG. 7. (Color online) Differential cross section for ${ }^{11} \mathrm{Be}(p, d){ }^{10} \mathrm{Be}$ transfer reactions at $E_{p}=35.3 \mathrm{MeV}$ leading to the ground $\left(0^{+}\right)$and excited $\left(2^{+}\right)$states of ${ }^{10} \mathrm{Be}$. CX predictions based on CH89 (solid curves), Koning-Delaroche (double-dotted-dashed curves), and Watson (dashed curves) potentials are compared with the experimental data from Ref. [33].

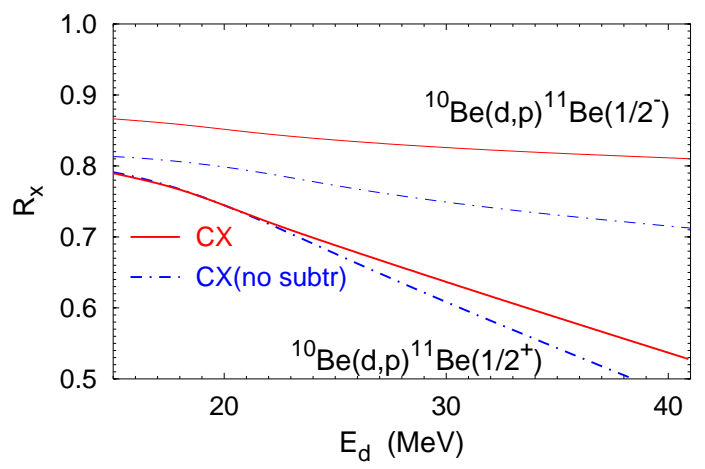

FIG. 8. (Color online) Differential cross sections ratios $R_{x}$ for ${ }^{10} \mathrm{Be}(d, p){ }^{11} \mathrm{Be}$ transfer reactions leading to ground (thick curves) and excited (thin curves) states of ${ }^{11} \mathrm{Be}$. CX and CX(no subtr) predictions are given by solid and dasheddotted curves, respectively.

is in contrast with naive assumptions often used for the SF extraction by comparing DWBA-type SP calculations and experimental data. In Fig. 8I confirm these findings of Ref. 19 also when using an improved potential with CeX. For $x$ being either CX or CX(no subtr) I show the ratios $R_{x}=(d \sigma / d \Omega)_{x} /(d \sigma / d \Omega)_{\mathrm{SP}}$ in $\left.{ }^{10} \mathrm{Be}(d, p)\right)^{11} \mathrm{Be}$ reactions at $\Theta_{\text {c.m. }}=0^{\circ}$ as functions of the deuteron energy $E_{d}$. The deviation of $R_{x}$ from the $\mathrm{SF}$, which equals to 0.855 (0.786) for the ${ }^{11} \mathrm{Be}$ ground (excited) state, becomes most evident for the transfer to the ${ }^{11} \mathrm{Be}$ ground state at higher $E_{d}$. Both CX and CX(no subtr) mod-
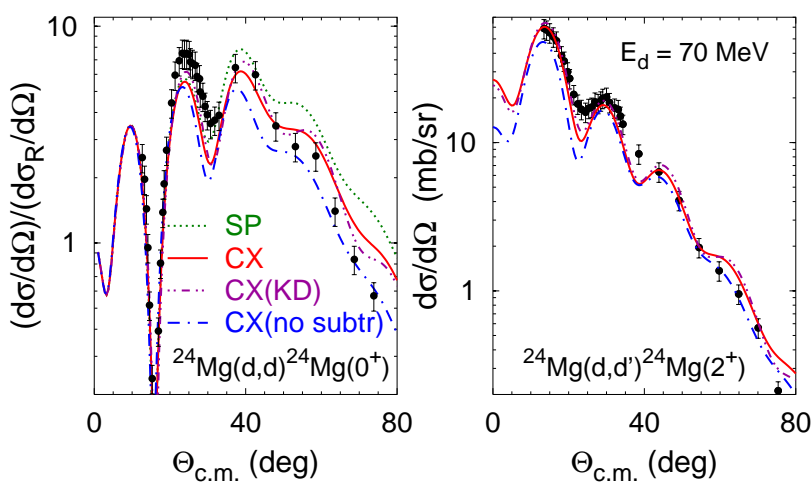

FIG. 9. (Color online) Differential cross section for $d+{ }^{24} \mathrm{Mg}$ elastic (left) and inelastic (right) scattering at $E_{d}=70$ $\mathrm{MeV}$. Results of SP, CX, and CX(no subtr) models based on the CH89 potential are given by dotted, solid, and dashed-dotted curves, respectively, while CX results based on the Koning-Delaroche potential are given by double-dotteddashed curves. The experimental data are from Ref. 34].

els show qualitatively the same behavior. The difference between them increases with increasing $E_{d}$ and thereby indicates the importance of the proper fit to the two-body data, but does not alter the general conclusion.

Finally I consider the $d+{ }^{24} \mathrm{Mg}$ scattering. The quadrupole deformation $\beta_{2}$ for ${ }^{24} \mathrm{Mg}$ determined in different experiments varies between 0.4 and 0.6 (see Ref. 34] for overview). In Ref. [19] the CX(no subtr) calculations were performed with $\beta_{2}=0.4$ and 0.6 , with the lower value favored by the ${ }^{24} \mathrm{Mg}(d, d)^{24} \mathrm{Mg}$ data and the upper value favored by the ${ }^{24} \mathrm{Mg}\left(d, d^{\prime}\right)^{24} \mathrm{Mg}^{*}$ data, but no one of them was able to reproduce the data for both reactions simultaneously. Here I present in Fig. 9 the results for $d+{ }^{24} \mathrm{Mg}$ elastic and inelastic differential cross section at $E_{d}=70 \mathrm{MeV}$ obtained with $\beta_{2}=0.5$. The CX model yields the best description of the experimental data and fits reasonably well both elastic and inelastic cross sections, while the $\mathrm{CX}$ (no subtr) model overestimates the CeX effect for the ${ }^{24} \mathrm{Mg}(d, d)^{24} \mathrm{Mg}$ but underestimates for the ${ }^{24} \mathrm{Mg}\left(d, d^{\prime}\right)^{24} \mathrm{Mg}^{*}$ reaction. Changing the starting potential from CH89 to Koning-Delaroche has only a small effect as demonstrated by the $\mathrm{CX}(\mathrm{KD})$ results.

\section{SUMMARY}

I considered elastic, inelastic, and transfer reactions in three-body nuclear systems consisting of a neutron, a proton, and a core. I explicitly included the core excitation in the exact scattering equations and solved them in the momentum-space framework. The calculational technique was taken over from Ref. [19] but the dynamic input was significantly improved.

The technical objective of this work was to develop a method for constructing the nucleon-core potential that couples ground and excited states of the core and 
in the coupled-channel two-body Lippmann-Schwinger equation reproduces the predictions of the given standard optical potential for the elastic scattering as well as the DWBA predictions for the inelastic reaction. The essence of the method is subtracting from the original singlechannel potential the explicit core excitation contributions, thereby avoiding the double counting. In the usual case where a coupled-channel potential properly fitted to the experimental data is not available but the nucleus deformation parameters are determined in the DWBA, the proposed method yields the coupled-channel potential including the core excitation and consistent with the data for both elastic and inelastic scattering. This is an important improvement over the simple deformation of the potential used previously [19] that destroys the fit to the experimental data. For the $p+{ }^{10} \mathrm{Be}$ example it is demonstrated that this deviation may be significant, especially for the inelastic $\left(p, p^{\prime}\right)$ reaction.

The physics objective of the present work was the evaluation of changes in three-body observables due to the improvement of employed optical potentials. In three-body reactions involving ${ }^{10} \mathrm{Be}$ and ${ }^{24} \mathrm{Mg}$ cores the most important changes were found in elastic $(p, p)$ and $(d, d)$, inelastic $\left(d, d^{\prime}\right)$, and transfer $(p, d)$ reactions leading to the core in its excited state. Transfer reactions $(d, p)$ were affected less, at least at lower energies. In $p+{ }^{11} \mathrm{Be}$ elastic scattering the core excitation effect turns out to be very small. Compared to results of Ref. [19], the improved nucleon-core potential leads to larger cross sections in most cases. This increases slightly the discrepancy between predictions and data for the ${ }^{10} \mathrm{Be}(d, p){ }^{11} \mathrm{Be}^{*}$ transfer reaction but improves the agreement for the ${ }^{11} \mathrm{Be}(p, d){ }^{10} \mathrm{Be}^{*}$ reaction. In the latter case I also found a significant sensitivity to the parametrization of the standard optical potential that serves as a starting point in the calculations. Concerning the $d+{ }^{24} \mathrm{Mg}$ scattering, the improved calculations are able to describe elastic and inelastic cross sections simultaneously with the same value of the deformation parameter $\beta_{2}=0.5$, in contrast to Ref. [19]. Finally, I qualitatively confirmed the findings of Ref. [19] that the effect of the core excitation in transfer reactions cannot be simply related to the respective spectroscopic factor.
[1] S. Quaglioni and P. Navratil, Phys. Rev. Lett. 101, 092501 (2008).

[2] L. D. Faddeev, Zh. Eksp. Teor. Fiz. 39, 1459 (1960) [Sov. Phys. JETP 12, 1014 (1961)].

[3] E. O. Alt, P. Grassberger, and W. Sandhas, Nucl. Phys. B2, 167 (1967).

[4] O. A. Yakubovsky, Yad. Fiz. 5, 1312 (1967) [Sov. J. Nucl. Phys. 5, 937 (1967)].

[5] P. Grassberger and W. Sandhas, Nucl. Phys. B2, 181 (1967); E. O. Alt, P. Grassberger, and W. Sandhas, JINR report No. E4-6688 (1972).

[6] A. Kievsky, M. Viviani, and S. Rosati, Phys. Rev. C 64, 024002 (2001).

[7] J. Kuros-Zolnierczuk, H. Witała, J. Golak, H. Kamada, A. Nogga, R. Skibiński, and W. Glöckle, Phys. Rev. C 66, 024004 (2002).

[8] A. Deltuva, A. C. Fonseca, and P. U. Sauer, Phys. Rev. Lett. 95, 092301 (2005).

[9] A. Deltuva, A. M. Moro, E. Cravo, F. M. Nunes, and A. C. Fonseca, Phys. Rev. C 76, 064602 (2007).

[10] R. Lazauskas, Phys. Rev. C 86, 044002 (2012).

[11] M. Viviani, L. Girlanda, A. Kievsky, and L. E. Marcucci, Phys. Rev. Lett. 111, 172302 (2013).

[12] A. Deltuva and A. C. Fonseca, Phys. Rev. Lett. 113, 102502 (2014).

[13] R. C. Johnson and P. J. R. Soper, Phys. Rev. C 1, 976 (1970).

[14] N. Austern, Y. Iseri, M. Kamimura, M. Kawai, G. Rawitscher, and M. Yahiro, Phys. Rep. 154, 125 (1987).

[15] D. Baye, P. Capel, P. Descouvemont, and Y. Suzuki, Phys. Rev. C 79, 024607 (2009).

[16] R. Crespo, A. Deltuva, and A. M. Moro, Phys. Rev. C
83, 044622 (2011).

[17] A. M. Moro and R. Crespo, Phys. Rev. C 85, 054613 (2012).

[18] A. M. Moro and J. A. Lay, Phys. Rev. Lett. 109, 232502 (2012)

[19] A. Deltuva, Phys. Rev. C 88, 011601(R) (2013).

[20] A. M. Mukhamedzhanov, V. Eremenko, and A. I. Sattarov, Phys. Rev. C 86, 034001 (2012).

[21] F. Nunes, J. Christley, I. Thompson, R. Johnson, and V. Efros, Nucl. Phys. A 609, 43 (1996).

[22] C. Hajduk, P. U. Sauer, and W. Strueve, Nucl. Phys. A405, 581 (1983).

[23] J. R. Taylor, Nuovo Cimento B 23, 313 (1974); M. D. Semon and J. R. Taylor, Nuovo Cimento A 26, 48 (1975).

[24] E. O. Alt and W. Sandhas, Phys. Rev. C 21, 1733 (1980).

[25] A. Deltuva, A. C. Fonseca, and P. U. Sauer, Phys. Rev. C 71, 054005 (2005).

[26] R. Machleidt, Phys. Rev. C 63, 024001 (2001).

[27] R. L. Varner, W. J. Thompson, T. L. McAbee, E. J. Ludwig, and T. B. Clegg, Phys. Rep. 201, 57 (1991).

[28] N. W. Schellingerhout, L. P. Kok, S. A. Coon, and R. M. Adam, Phys. Rev. C 48, 2714 (1993).

[29] B. A. Watson, P. P. Singh, and R. E. Segel, Phys. Rev. 182, 977 (1969).

[30] A. J. Koning and J. P. Delaroche, Nucl. Phys. A713, 231 (2003).

[31] V. Lapoux et al., Phys. Lett. B 658, 198 (2007).

[32] K. T. Schmitt et al., Phys. Rev. Lett. 108, 192701 (2012).

[33] J. Winfield et al., Nucl. Phys. A683, 48 (2001).

[34] A. Kiss, O. Aspelund, G. Hrehuss, K. Knopfle, M. Rogge, U. Schwinn, Z. Seres, P. Turek, and C. Mayer-Boricke, Nucl. Phys. A 262, 1 (1976). 